\title{
Ropa tendida: Gestos de la experiencia cotidiana de la ciudad
}

Recibido: 9 de octubre 2019 Revisado: 24 de febrero 2020

Aprobado: 3 de marzo 2020

Rebeca Silva Roquefort Chilena. Doctora en Arquitectura y Urbanismo, Universidad Politécnica de Madrid, España y diplomada

en Estudios Avanzados en

Urbanística y Ordenamiento

Territorial. Diplomada de

Postítulo en Ergonomía, Universidad de Chile, Chile. Labora en la Universidad de Chile, Chile. Correo electrónico: bekysilva@uchilefau.cl

Luis Campos Medina

Chileno. Sociólogo chileno de la Universidad de Chile. Máster en Ciencias Sociales y doctor en Sociología por la École des Hautes Études en Sciences Sociales, Francia . Académico e investigador del Instituto de la Vivienda de la Universidad de Chile, Chile. Correo electrónico: luiscampos@uchilefau.cl

Josefina Jaureguiberry Mondion

Chilena. Cientista política de la Pontificia Universidad Católica de Chile, Chile. Magíster en Estudios

Urbanos de la University

College London, Londres, Reino Unido, y actual estudiante de doctorado en Geografía y medioambiente en la University of Oxford, Reino Unido. Correo electrónico: josefina.jaureguiberry@chch. ox.ac.uk
Resumen: Por obvia e insignificante, la ropa tendida pasa la mayor parte del tiempo desapercibida en nuestro deambular cotidiano. Sin embargo, asomada por las ventanas o flotando con el viento, es parte del paisaje urbano de nuestras ciudades. Más aún, en su supuesta insignificancia, la ropa tendida sugiere múltiples aspectos de la vida actual: restricciones e inadecuaciones del espacio; rutinas laborales y de descanso; identidades sociales y coordinaciones comunitarias. ¿Qué vemos cuando vemos la ropa tendida? ¿Seguimos viendo nuestra vestimenta? ¿O comenzamos a ver colores, formas, historias, intimidades? Des-guardar y exponer nuestra vestimenta puede interpelarnos en varios registros: ¿qué es lo que decidimos exponer? ¿Qué es legítimo hacer visible? ¿Qué cosas escondemos del escrutinio de los otros? En este texto, indagamos sobre cómo la presencia de la ropa tendida puede procesarse como una serie de gestos, las cuales remiten a diferentes acercamientos a las materialidades que conforman nuestra experiencia cotidiana de la ciudad. Estos vinculan lo funcional con lo poético y lo expuesto con lo íntimo. Inspirados en la noción de "lo infraordinario" de Perec, y apoyándonos en la comprensión de la importancia de los gestos cotidianos y la sociología de los objetos, exploramos la potencia sensible, pública y política de la ropa tendida en entornos urbanos.

Palabras clave: ropa tendida; infraordinario; gestos; producción del espacio

\section{Hangin Clothes: Gestures of the Everyday Experience of the City}

Abstract: Obvious and insignificant, hanging clothes spend most of the time unnoticed in our daily wanderings. However, leaning out of the windows or floating in the wind, they are part of the urban landscape of our cities. Moreover, in its supposed insignificance, hanging clothes suggest multiple aspects of current life: restrictions and inadequacies of space; work and rest routines; social identities and community coordinations. What do we see when we see hanging clothes? Do we still see our clothes? Or do we begin to see colors, shapes, stories, intimacies? Des-save and expose our clothes can interpellate us in several ways: what do we decide to expose? What is it legitimate to make visible? What things do we hide from the scrutiny of others? In this text we inquire about how the presence of hanging clothes can be understood as a series of gestures that refer to different approaches to the materialities that make up our daily experience of the city. Gestures that link the functional with the poetic and exposed with the intimate. Inspired by the notion of Perec's "infraordinary" and based on the understanding of the importance of everyday gestures and the sociology of objects, we explore the sensitive, public and political power of hanging clothes in urban environments.

Key words: hanging clothes; infraordinary; gestures; production of space 


\section{Introducción}

En el texto que abre el libro Lo Infraordinario, Georges Perec $(2008,21)$ expresa su profundo desacuerdo con el modo en que actualmente nos acercamos y construimos el mundo, basándonos en los acontecimientos grandilocuentes: "...como si la vida no debiera revelarse nada más que a través de lo espectacular, como si lo elocuente, lo significativo fuese siempre anormal...". La postura de Perec es completamente la opuesta: encontrar la vida en lo miserablemente excepcional, en aquello que pasa completamente desapercibido, pero que nos hace ser quienes somos y constituye nuestro diario vivir. Por ello el escritor presta una atención poco usual a los gestos, a la procedencia, el uso y el devenir de los objetos de nuestras vidas cotidianas. Su apuesta literaria e intelectual gira alrededor de la idea de que la vida se juega en los detalles, en una coreografía de pequeños y sutiles gestos. Perec nos conmina a interrogarnos por lo habitual, reconociendo que nuestras existencias y verdades funcionan a través de sus particularidades.

Al enfrentarse con la propuesta de Perec, resulta inevitable preguntarse por la relación existente entre lo particular y lo general -la vinculación del detalle con la totalidad-. En la propuesta de Perec, podemos encontrar, constantemente, intentos de indagar cómo los detalles son significativos dentro de un mundo social más amplio. En este sentido, su trabajo se encuentra fuertemente influenciado por el filósofo y sociólogo marxista Henri Lefebvre. En 1958, mientras Perec realizaba el servicio militar como paracaidista en el suroeste de Francia, se hospedó en la casa de Lefebvre (Highmore 2018, 109). Es justamente en esta época cuando se acababa de publicar la segunda edición de la Crítica de la vida cotidiana, en que Lefebvre desarrolla un análisis de las contradicciones y potencialidades de la vida moderna, haciendo hincapié en los detalles triviales de la experiencia cotidiana como fuente de resistencia y cambio. De esta manera, para Lefebvre, lo general sigue siendo importante, pero la forma de acceder a aquello es a través de los detalles y lo cotidiano. La influencia que tuvo Lefebvre en el trabajo de Perec se materializa en el desarrollo del concepto de lo infraordinario y la idea de que lo insignificante nos proporciona claves y pistas; una heurística para explorar nuestros mundos.

Pensar en lo cotidiano nos remite ineludiblemente al trabajo de Michel de Certeau. En su libro La invención de lo cotidiano (1979), el autor hace un llamado a la necesidad de prestar particular atención a las prácticas que constituyen nuestro día a día. De Certeau profundiza en la importancia de los gestos cotidianos y el potencial de emancipación que estos tienen. A través de su énfasis en el anonimato y lo mundano, lo cotidiano nos muestra el "reino fenomenológico esencial de lo vivido" (Courpasson 2017, 845). En diálogo con Perec y Lefebvre, de Certeau nos llama a pensar sobre cómo el significado se construye en nuestras vidas en torno a aquellas ocupaciones ordinarias (caminar al trabajo, cocinar la cena, tender la ropa) que suelen pasar 
desapercibidas (Garfinkel 1967, 226). Es justamente aquí donde nos interesa indagar: en los ritos nimios de nuestra vida cotidiana. Siguiendo esa inspiración, inicialmente pereciana, la ropa tendida dejó de ser evidente y se convirtió en objeto de nuestro interés; pasó de ser una entidad naturalizada y banal a convertirse en un objeto de atención sobre el cual volcar nuestras inquietudes y nuestra curiosidad. En este sentido, consideramos fundamental la reivindicación de los objetos de estudio "no prescritos", investigando aquellos temas y fenómenos que suelen estar fuera del repertorio de lo pensable y se consideran poco legítimos o no suficientemente importantes como para prestarles atención.

El hincapié en lo cotidiano de los antedichos pensadores franceses tiene su correlato actual en el llamado -que ha adquirido fuerza en el último tiempo en la disciplina sociológica- sobre la necesidad de centrarnos en los detalles y lo sucesos del día a día. Un representante eminente de ello es Les Back. Para este autor, la relevancia actual de las ciencias sociales se juega en su capacidad de prestar atención a detalles pasados por alto, a la vida oculta de objetos y lugares que se vuelven escurridizos por los efectos del poder, del olvido o de las convenciones. Las ciencias sociales tienen aquí una tarea crucial, pero también un importante desafío epistemológico, puesto que hablar de lugares, objetos e individuos vueltos invisibles como resultado de nuestra curiosidad adormecida, nuestras rutinas analíticas y nuestras economías de pensamiento no es una tarea sencilla. Requiere de imaginación (la "imaginación sociológica" de C. Wright Mills 1959) y de avanzar en una "democracia de los sentidos" (Back 2015, 8), que nos habilite a proponer nuevas perspectivas e interrogantes. En efecto, si volvemos a Perec, nos encontramos con una serie de preguntas claras y directas respecto del desafío que involucra describir ese mundo cotidiano y oculto: "Lo que realmente ocurre, lo que vivimos, lo demás, todo lo demás, ¿dónde está? Lo que ocurre cada día y vuelve cada día, lo trivial, lo cotidiano, lo evidente, lo común, lo ordinario, lo infraordinario, el ruido de fondo, lo habitual, ¿cómo dar cuenta de ello? ¿cómo interrogarlo? ¿cómo describirlo? (Perec 2008, 22-23)”.

\section{Lo cotidiano como una coreografía de gestos}

El cambio de perspectiva recién indicado implica problematizar lo evidente, identificar síntomas e interrogar las huellas de procesos mayores. La ropa tendida es una expresión, entre muchas posibles, de ese afán. Esta inspiración "pereciana" ha entrado en conversación con otras perspectivas de pensamiento y se ha convertido en un programa de investigación dirigido a interrogar lo trivial, lo nimio y los aspectos más prosaicos de nuestra experiencia. Entre esas perspectivas, se encuentra el programa de investigación que las ciencias sociales heredan de la literatura. Según Jacques Rancière (2009), se trata de encontrar los síntomas de algo mayor, hasta en los más mínimos detalles de la vida ordinaria: 
Pasar de los grandes acontecimientos y personajes a la vida de los seres anónimos, encontrar los síntomas de una época, una sociedad o una civilización en los detalles ínfimos de la vida corriente, explicar la superficie a través de las capas subterráneas y reconstituir mundos a partir de sus vestigios, este programa es literario antes que científico. Esto no significa solamente que la ciencia histórica tenga una prehistoria literaria. Es la literatura misma la que se constituye como una cierta sintomatología de la sociedad y opone esta sintomatología a los gritos y a las ficciones de la escena pública (Rancière 2009, 11).

En este sentido, el concepto de síntoma mencionado por Rancière resuena al llamado de Perec de reconocer que lo general solo puede funcionar en sus particularidades. Ambas propuestas parten de una misma constelación: aquella que nos quiere mostrar que nuestra humanidad se ha constituido sobre la base de movimientos pequeños, de sutiles ajustes que dan forma a nuestras existencias.

Cabe mencionar aquí que la pregunta por la vida cotidiana ha sido asumida seriamente por medio del uso de métodos etnográficos en diferentes frentes de las ciencias sociales (Pink 2012, 7). Fuera de la ya nombrada triada de pensadores franceses, Perec, Lefebvre y De Certeau, el trabajo del sociólogo Ervin Goffman (1959), la geógrafa Doreen Massey (2005) y el antropólogo Tim Ingold (2011) constituyen solo algunos ejemplos notables de trabajos que se han encargado de estudiar aquello que aparentemente carece de importancia. Desde diferentes disciplinas se ha constituido una agenda de trabajo que cuestiona la "textura política de la vida cotidiana" (Courpasson 2017, 847) y plantea la oportunidad de vincular los pequeños relatos de nuestros días con grandes historias y posibles transformaciones sociales de mayor alcance.

En esta línea, Sheringham $(2000,188)$ es hábil al describir lo cotidiano como aquello "superficial y profundo, extraño y familiar, insignificante y fundamental, fuera de la praxis y sin embargo el presagio de las energías anárquicas". Es aquí donde situamos nuestro interés por la ropa tendida, en el afán por comprender las capas de significado que podemos encontrar si prestamos atención a sus detalles. De esta forma, a través de la identificación de los diferentes gestos de la ropa tendida, esperamos acercarnos a comprensiones más amplias de nuestra vida cotidiana.

No obstante, antes de pasar al análisis de la ropa tendida, consideramos pertinente indagar en la decisión de adoptar el concepto de "gesto". Los gestos se asocian, usualmente, a modos de comunicación no verbal que toman for- 
ma a través de una determinada corporalidad o movimiento. De esta manera, han sido descritos como una forma de inscribir prácticas culturales específicas en el cuerpo (Noland 2009, 2). Es decir, los gestos son el resultado del aprendizaje corporal de patrones de comunicación no verbal. Sin embargo, pareciera que el gesto va más allá y es capaz de escapar a la concepción que lo entiende como contenido en el cuerpo humano y como resultado de una función práctica y orientada a un objetivo o meta.

En esta línea, los gestos han sido pensados a través de la capacidad que tienen para contener significado y poder expresarlo. En su libro Gestures, Vilém Flusser (2014) define al gesto como un movimiento del cuerpo, o de una herramienta conectada al cuerpo, el cual es simbólico y requiere que su significado sea descubierto en su especificidad. El ejercicio al que nos invita Flusser es el de prestar atención a la manera en que estos movimientos son capaces de estructurar significado más allá de su función práctica. El gesto, en cuanto portador y a la vez productor de significado, llama la atención de quien se encuentra con él: es una invitación a develar un significado. En este sentido, los gestos conectan cuerpos y también objetos, siendo capaces de afectarnos a través del encuentro y la transmisión de ese significado y simbolismo.

Es importante resaltar que, en la concepción de gestos aquí desarrollada, estos no necesariamente están vinculados únicamente al cuerpo humano, sino que también pueden "establecer una continuidad entre materialidad e inmaterialidad" y sobrepasar el gesto contenido en el agente humano (Bardet 2018, 20). A pesar de que el cuerpo no está presente, existen elementos que contienen significado y están relacionados con el o los cuerpos que, en primera instancia, lo posicionaron. Esta comprensión de los gestos plantea que objetos inmateriales pueden ser contenedores de significado. De esta forma, los gestos no se limitan a su humanidad, pueden existir en el flujo entre componentes humanos y no humanos. Es el caso de la ropa tendida. Resultante de un accionar humano, una vez que se encuentra flotando en los recovecos urbanos, deviene en gesto vinculado al cuerpo que la colgó, pero trascendiéndolo y contribuyendo a componer la coreografía de gestos de nuestra vida cotidiana, tal como nos recuerda Lefebvre, con su noción de ritmoanálisis, según la cual la vida cotidiana es una sucesión armónica de ritmos (Lefebvre y Régulier 1985).

Con esta comprensión de gesto que hemos desarrollado aquí, aparecen una serie de preguntas que podemos plantearle a la ropa tendida. ¿De qué manera sigue el cuerpo presente en la ropa colgada? ¿Cómo se manifiestan nociones más amplias de nuestro habitar a través de nuestra ropa tendida? ¿Qué nos dice la ropa flotante, limpia y colorida, en medio de los grises de nuestras ciudades? Podríamos afirmar que la ropa al viento contiene un cuerpo invisible y que el cuerpo sigue viviendo a través de ella. No es el cuerpo en sí mismo, pero nos remite a él constantemente. A continuación, indagaremos en la ropa tendida en cuanto gesto que se torna huella y manifestación de quehaceres materiales, corporales, sociales, políticos, poéticos y paisajísticos. 


\section{Los múltiples gestos de la ropa tendida}

Los estudios actuales de la vestimenta se han centrado en el rol que esta juega en la mercantilización de las culturas. Diferentes análisis antropológicos han estudiado cómo la ropa, fabricada y comercializada, simboliza identidades sociales y políticas (Bayly 1986; Weiner y Schneider 1989). Estos estudios han puesto el foco en cómo las prendas de vestir han adquirido nuevos valores en un contexto de relaciones de mercado (Margiotti 2013, 389). A la vez, se ha hecho un llamado de atención que invita a estudiar la vestimenta en términos de su capacidad para mediar relaciones entre personas y su capacidad para materializar valores y estilos de sociabilidad. El llamado a estudiar la "vida social de las cosas" (Appadurai 1986) se ha traducido en estudios sobre la ropa como mediador de identidades (Banerjee y Miller 2003; Küchler y Miller 2005) y una variedad de temas que van desde cómo las personas se deshacen de las prendas en desuso (Norris 2004) hasta la estructuración de identidades de género a través de cómo nos vestimos (Gregson y Beale 2004). El renovado enfoque dentro de las ciencias sociales por la materialidad de nuestra existencia ha redundado en una serie de estudios sobre la ropa que retoma la idea de que esta tiene un ciclo de vida en sí misma, el cual está a su vez arraigado en la vida de quienes la usan y descartan.

A pesar de este interés por la ropa, no existe ningún estudio que se centre específicamente en cómo colgamos nuestra ropa, ni en los efectos de la ropa tendida en lo urbano. Aunque es una práctica profundamente encarnada en nuestras vidas, pareciera ser que su misma cotidianidad desalienta cualquier interés por prestarle atención. Según argumentamos anteriormente, la ropa se constituye como una serie de gestos que encarnan formas de hacer y que, en tanto huellas significantes, nos permiten acceder a comprensiones más amplias de nuestras vidas y de nuestros mundos. A continuación, describiremos una serie de gestos que se pueden identificar al prestar atención a la ropa tendida. Los gestos que describiremos no pretenden ser exhaustivos, por el contrario, buscan abrir la puerta a otras formas de comprender este acto doméstico.

\section{Gesto material: adaptar y producir}

La planificación y diseño de la ciudad, en sus formas más tradicionales, ocurren sobre la base de aquellos usos esperados del espacio, reduciéndose a lo visible y legible que sucede en las ciudades (Martínez 2013). De esta manera, una parte importante de las prácticas que producen diariamente el espacio quedan invisibilizadas o relegadas a un segundo plano. Sin embargo, como se puede ver a través de la ropa tendida colgada en distintos lugares, nuestras ciudades están llenas de usos inesperados del espacio que producen una realidad material mutable y alternativa. En este sentido, queremos recalcar la noción de la ropa tendida como un gesto material que simboliza una forma específica de habitar el espacio. Un gesto que nos remite a las condiciones en las que viven diferentes sectores de la población, los cuales 
deben recurrir al ingenio y a la inventiva para solucionar problemáticas de la vida cotidiana. La ropa tendida nos habla de una forma de habitar el espacio que está en constante tensión con los usos preestablecidos del espacio. Este gesto material, a su vez, es solo un botón de muestra de una realidad mayor relacionada con la manera en que las personas construyen ciudad por medio de las pequeñas prácticas cotidianas que disputan los usos del espacio cuando la planificación y la política urbana son incapaces de dar respuesta a sus necesidades.

La figura 1 corresponde a una fotografía tomada desde abajo, en un block "tipo C" de tres pisos de ladrillos rojos. En el acceso al primer piso, se ve una reja de madera que deslinda con la calle. Sobre la baranda enrejada del tercer piso cuelga la ropa tendida frente a los departamentos. La ropa tendida se encuentra en el centro de la fotografía y convive con una variedad de elementos: desde plantas hasta bicicletas, pasando por luces de decoración, toldos y antenas de televisión. Arriba, el cielo azul es entrecortado por salpicadas nubes blancas. En esta fotografía, la ropa tendida sugiere la inexistencia de espacios interiores específicos para colgar ropa, pero también informa de la multiplicidad de nuevos usos del espacio que desbordan el programa original, de la maleabilidad de los entornos construidos y de la adaptabilidad e inventiva de quienes ahí habitan.

Figura 1. Avenida Punta Arenas, Comuna de La Florida. Enero 2019

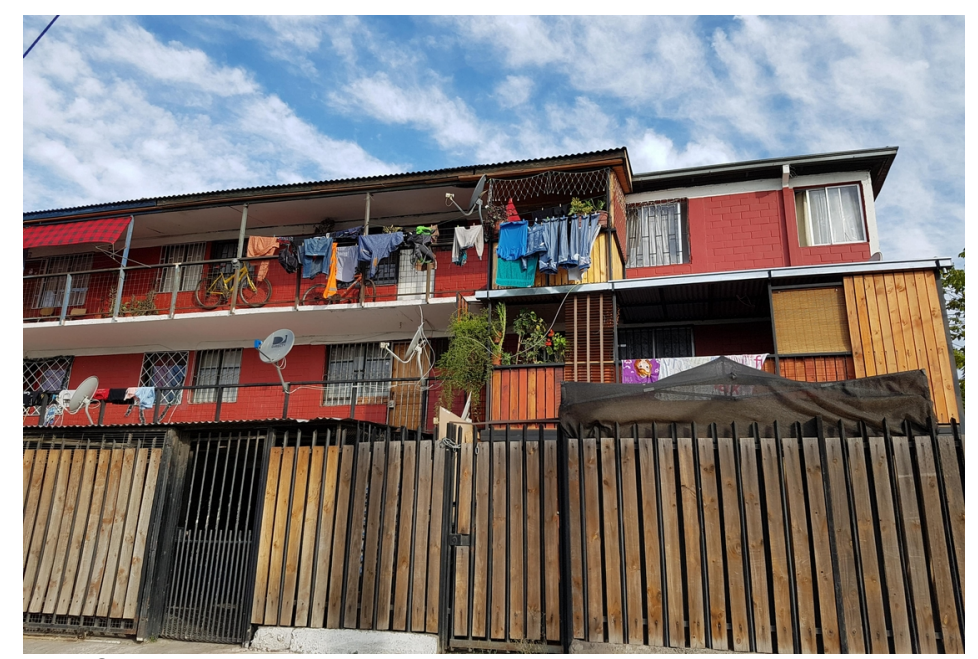

Fuente: Silva (2019)

En su aspecto material, colgar la ropa es primordialmente usar un espacio para secar la ropa recién lavada, siendo la manera más económica para suplir la carencia de otros medios para hacerlo (una secadora eléctrica, por ejemplo). Muchas veces, las viviendas no cuentan con un espacio destinado a esta tarea y los residentes deben buscar soluciones alternativas que suelen estar guiadas por el ingenio. Esta apropiación del espacio, en función de las necesidades de los residentes, se ha denominado estrategias adaptativas (Silva, Muñoz y Delanoe 2016; Hernández García, Bernal y Hernández-Gar- 
cía 2018). Estas estrategias suelen emerger en contextos donde las viviendas no resuelven de manera adecuada las necesidades y usos de las personas que en ellas residen, afectando así sus condiciones de habitabilidad. En la literatura sobre asentamientos informales, esta forma de resolver necesidades cotidianas ha sido comúnmente explicada en términos de carencia e irregularidad (Hernández García, Bernal y Hernández-García 2018, 127). Sin embargo, recientemente, y buscando resaltar las contribuciones positivas de estos "sistemas de adaptación" (Ibid.), diferentes autores han argumentado a favor de entender cómo estas se constituyen como formas alternativas de pensar y gestionar las condiciones socioespaciales de las ciudades (Roy 2009; Robinson 2006). Para efectos de este artículo, la ropa tendida, en cuanto estrategia adaptativa, emerge como resultado de una necesidad y toma forma mediante una intervención material. A su vez, la ropa tendida tiene una repercusión en el espacio y en las formas de vida de los habitantes del lugar, pues, en su emergencia, es capaz de producir realidades dentro del espacio del conjunto habitacional y la comunidad.

Este gesto material tiene, además, otra arista relacionada a una dinámica interna de la producción del espacio dentro del vecindario o barrio. El despliegue de tal estrategia adaptativa puede estar sustentada en la articulación y coordinación entre vecinos y en la construcción de cierta noción de comunidad. Por ejemplo, cuando la ropa se cuelga en las azoteas de los edificios, los vecinos deben organizarse y establecer ciertos códigos de conducta que regulan explícita o implícitamente el uso del espacio. A la vez, colgar la ropa limpia en espacios comunes puede derivar en dinámicas de conflicto y competencia dentro de la colectividad que debe gestionar ese espacio. En cualquiera de los dos casos, a través de la ropa tendida podemos acercarnos a maneras más amplias de gestión y producción del espacio, y a ver cómo esto se relaciona con las dinámicas internas de la comunidad propietaria de la vestimenta. La ropa tendida se vuelve un gesto que encarna las relaciones sociales y materiales que constituyen las prácticas rutinarias de la vida cotidiana (Dant 1999, 61) y colectiva.

Encontramos aquí una suerte de resonancia de los planteamientos de Hernández García (2013) en la medida que la materialidad observada "corresponde largamente a las necesidades, expectativas, posibilidades y construcciones simbólicas de los habitantes" (Hernández García 2013, 143). Las prácticas se modifican para acomodarse a las interacciones con el espacio, mientras que ese espacio muta para adaptarse a las prácticas de quienes lo habitan. Se produce, entonces, una materialidad que da cuenta de interacciones y enuncia significados.

\section{Gesto político: visibilizar rompiendo con los binarios}

Como hemos indicado en el punto anterior, la "acción insignificante" de colgar la ropa construye significado y comunidad por medio de su intervención material del espacio (Courpasson 2017). Esta comprensión de lo insignificante le otorga un papel profundamente político, ya que, a través de estos detalles, se refuerzan o disputan formas de hacer mundo. De acuerdo con esto conside- 
ramos que la ropa tendida puede ser vista como un gesto político al menos en dos sentidos. Por un lado, nos hace cuestionarnos sobre la relación entre lo íntimo y lo público, y cómo esta práctica genera quiebres en la tradicional distinción público-privado. Por otro lado, nos permite problematizar la distinción de clases como operación de relegación e invisibilización, por lo que posibilita preguntarnos por cómo se produce el confinamiento de determinadas prácticas en ciertos sectores de la ciudad.

Figura 2. Exequiel Fernández, Comuna de Macul. Agosto 2016

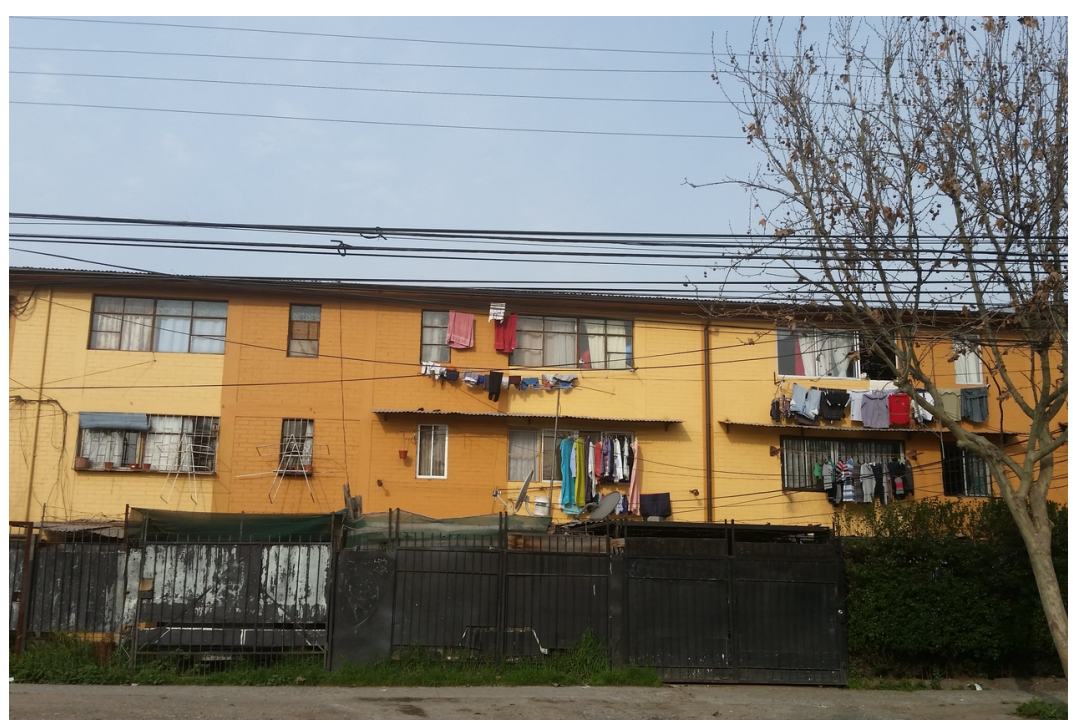

Fuente: Silva (2016)

La figura 2 muestra un conjunto habitacional de tres pisos cuya fachada ha sido recientemente pintada; el cielo y las hojas de un árbol situado a la derecha de la imagen sugieren un día invernal. Desde las ventanas del tercer y el segundo piso cuelgan cables con ropa tendida; en el tercer piso, la ropa se observa colgada directamente en el cable; en el segundo, la ropa está dispuesta en perchas sujetas a los cables. En un par de ventanas del segundo piso no hay ropa tendida, pero se aprecia la instalación de colgadores metálicos, vale decir, estructuras diseñadas para colgar la ropa en espacios reducidos y generalmente en el interior de las viviendas, pero aquí se encuentran instalados directamente en la estructura de las ventanas.

Este aspecto conecta con lo indicado en la figura 1, pero nos hace avanzar en la idea de una prolongación del espacio interior hacia el exterior, en una intervención de la espacialidad de las viviendas que interfiere la distinción canónica entre público y privado. Más aún, la imagen muestra la existencia de antejardines en el perímetro de los edificios, los cuales están delimitados por sendas rejas negras y metálicas. Incluso, se insinúan algunos techos en dichos antejardines. La ropa siempre está dentro de ese perímetro, salvo que conecta visualmente con cualquier transeúnte o automovilista que circule por esta concurrida calle, como en el caso de la autora de la fotografía. 
La ropa tendida es capaz de generar quiebres en el binario público/privado. Esto lo hace en dos niveles: primero, visibilizando aspectos supuestamente íntimos de nuestra existencia, rompe con el pudor de mostrarlos; segundo, cuestiona las maneras del uso del espacio público a través de lo que anteriormente denominamos como estrategias adaptativas, puesto que, debido a la necesidad de solucionar un problema práctico de la vida cotidiana y doméstica, se utiliza el espacio como un continuo. Entonces, ya no hay distinción entre adentro y afuera, sino una solución que, en vez de preguntarse por las convenciones del espacio, opta por ejercer la practicidad ingeniosa del "suple" y de las extensiones. Estos escapes a la distinción público/privado son, en otras palabras, el resultado del rasgo más básico de colgar la ropa: el exponer la ropa lavada, sacarla a la luz y hacerla visible.

\section{Figura 3: Comuna de EI Monte. Enero 2018}

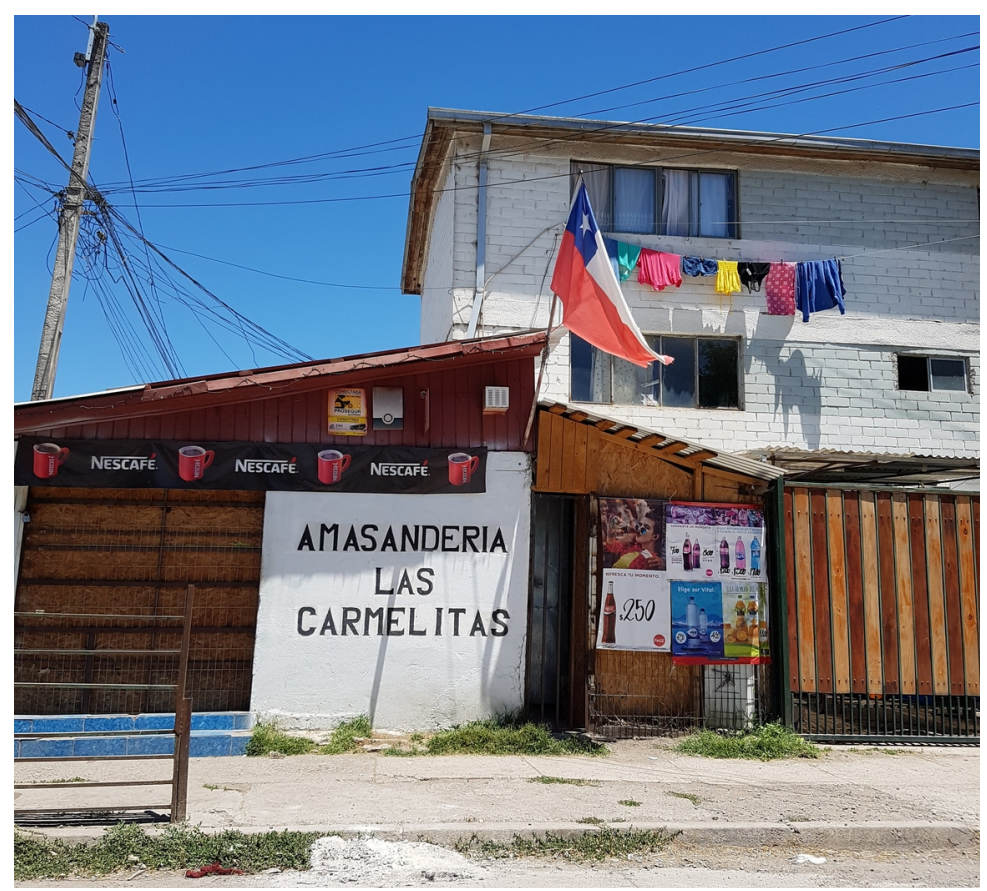

Fuente: Silva (2018).

En la figura 3, se observa un block blanco de tres pisos: en el primero, hay una ampliación destinada al comercio. En la pared, se lee "Amasandería las Carmelitas" en mayúsculas negras. Bajo la ventana del tercer piso, la ropa tendida ondea al viento, al igual que la bandera chilena a su lado, en el centro de la imagen. Arriba, a la izquierda, un poste de luz y una maraña de cables eléctricos. De fondo, el cielo azul. La imagen es elocuente: bandera chilena y ropa tendida aparecen en igualdad de condiciones; similares alturas, similares posiciones, similares posibilidades de visibilidad. En tiempos de expresión de identidad nacional, esta convivencia de ropa y bandera nos lleva a preguntarnos por el estatus de vehículo de identidad de la primera y por la densidad de significado que puede alcanzar. 
Si prestamos atención, en la medida en que la convención representacional tiende a retratarla como la posibilidad de movimiento absoluto (casi al modo del flamear de una bandera), la ropa tendida aparece como horizonte utópico. Además, sugiere un espacio propio y libre, cuestiones restringidas para los sectores populares. Tal vez, quienes cuelgan la ropa en espacios visibles para los transeúntes o para el vecindario no se plantean tender la ropa como un acto reivindicativo -al menos no de forma consciente-. Probablemente tiene más que ver con un accionar práctico: es la manera más eficaz de secar la ropa, sobre todo en contextos de escasez de espacio y poca luz solar al interior de la vivienda. Sin embargo, una vez allí, la ropa deviene signo de dignidad y de reivindicación. La ropa encarna el derecho a aparecer en el espacio y flotando, limpia y al viento, remite a valores relacionados a la pulcritud y la limpieza. Las personas deciden hacer visible aquello que es íntimo, pero solo cuando se encuentra en un estado de dignidad particular que le otorga la limpieza; la ropa tendida es, bajo esta perspectiva, un operador de reivindicación. Al afirmar esto, le asignamos una forma de agencia específica a la ropa tendida: depositando un cierto sentido en esta imagen se pretende, por delegación, que actúe sobre otros -sobre quienes la ven-, e intervenga sobre los significados que tales observadores asignan a los grupos sociales en cuestión.

Hay aquí una resonancia profunda con la reflexión desarrollada por Rancière en cuanto a la dimensión política del reparto de lo sensible (2009), puesto que esta noción justamente apunta a mostrarnos la relevancia que tienen las evidencias sensibles en la configuración de nuestro mundo y en la conformación de las delimitaciones que organizan nuestra percepción: la ropa tendida interpela el binarismo público/privado, así como las connotaciones de género que trae consigo; la ropa tendida se entrelaza con el símbolo patrio y aparece a los ojos del transeúnte con similares capacidades de simbolización y, así, como dice Rancière, interviene sobre "las maneras de hacer y sus relaciones con maneras de ser y formas de visibilidad" $(2009,11)$.

\section{Gesto poético y la construcción de imaginarios paisajísticos}

En la imagen 4, se ve una fachada continua de la tipología constructiva tradicional porteña. Rojo, azul, amarillo y techos color café cinc oxidado. Múltiples ventanas y abajo, a la izquierda, el acceso a una vivienda. Colgando de algunas ventanas, se ve la ropa tendida que compone el paisaje. Con esta imagen, queremos precisar esa asociación que realizamos inconscientemente entre las fachadas pintadas de colores y la ropa tendida; pues, más que visibilizar desigualdades, precariedad constructiva e incluso obsolescencia, aparece como un relato paisajístico de nuestras memorias de Valparaíso, transformándose en una suerte de postal de los cerros porteños. 
Figura 4. Cerro Bellavista, Valparaíso. Octubre 2016

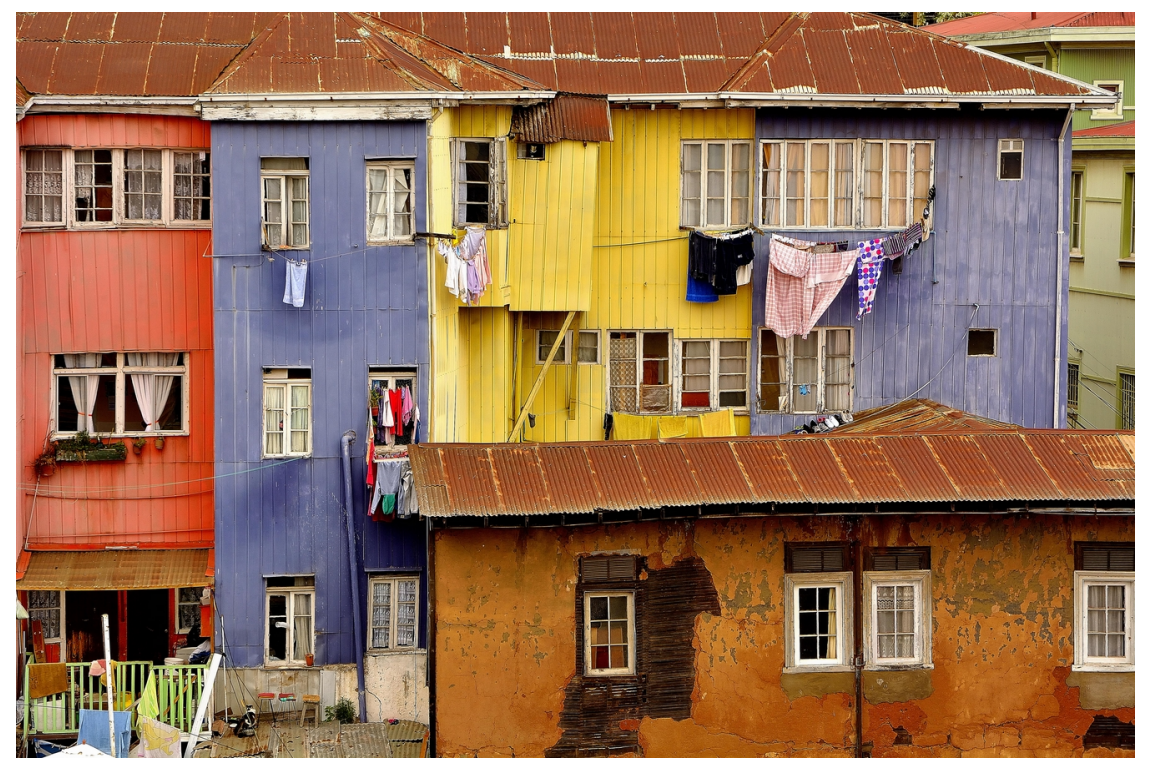

Fuente: Riquelme (2016).

En el acto de exponer nuestra ropa al sol y al viento, se juega ya con un elemento poético, un potencial de movimiento y color que exalta la labor efectuada: la ropa tendida es, en principio, ropa limpia, es decir, ropa que ha experimentado el trabajo humano; pero se trata de un trabajo específicamente dirigido a eliminar la suciedad, a apartar lo indeseable. En ese sentido, es un gesto estético en tanto productor de una actitud contemplativa de la belleza asociada a la pulcritud y la limpieza. Diferentes autores han estudiado cómo se construyen determinadas identidades de clase a través de la decoración de la vivienda, demostrando que, en sectores populares, existe una preferencia por elementos decorativos que parezcan nuevos, limpios y brillantes (Gullestad 1995; Miller 2005). Algo similar se puede encontrar al observar cómo es que la ropa encarna los valores estéticos de sus dueños. Se puede identificar una apreciación de la belleza del trabajo, de su contemplación y una estética del esfuerzo y los valores asociados a este.

La ropa colorida flotando al viento pareciera ser una instalación artística; una gama de colores que nos invita, a partir de su contemplación, a adentrarnos en las vidas íntimas de sus dueños. Es en este sentido que, abundante y reiterativa, la ropa tendida modula nuestra forma de percepción del entorno hacia una expectativa de colorido y limpieza que interviene en la monotonía y la monocromía de grises, activando los valores antes sugeridos: dignidad, trabajo, esfuerzo, mérito, superación, entre otros. La ubicuidad de la ropa tendida en las ciudades construye un imaginario paisajístico, algo a lo que estamos acostumbrados y que inconscientemente esperamos ver. Al caminar por las calles de Valparaíso, por ejemplo, de alguna manera esperamos ver la ropa tendida, siendo esta una imagen de la ciudad que se ha ido calcificando 
en nuestro imaginario de ciudad y, en su reiteración, generando una expectativa. De esta manera, la ropa tendida encarna una identidad que vas más allá de sus dueños, y nos permite ver, a través suyo, identidades citadinas.

¿Qué es lo primero que asalta nuestra mente cuando nombramos la ropa tendida? Para muchos, seguramente, aparecerán imágenes de diferentes ciudades donde pudieron observar ropa colgando de ventanas, balcones e incluso de techos. Pareciera ser algo que es parte del imaginario de diferentes paisajes urbanos. En sintonía con la discusión sobre imaginarios urbanos desarrollada en América Latina por Silva (1992), la ropa tendida forma parte de nuestra comprensión de ciudad y por medio de su repetición va reforzando una imagen de ciudad en la que ella está presente. Al ser vista por el transeúnte, la ropa colgada va retroalimentando la idea de que es parte de la urbe y va asentando la expectativa de ser encontrada y vista.

Cabe aquí convocar el reciente planteamiento de Rancière (2020) en relación con el paisaje de acuerdo con el cual este es mucho más que un simple espectáculo que captura nuestra atención visual y nos otorga goce espiritual. El paisaje es una forma que otorga unidad a la diversidad sensible y que, muchas veces, interpela las reglas perceptuales y las normas de apreciación estética; que contribuye a conformar, pero también, en ocasiones, cuestiona nuestras formas de representación identitaria y comunitaria. En este sentido, contemplar el paisaje muchas veces viene acompañado de una incitación a la acción, una búsqueda experiencial que combina y sintetiza estética y política.

\section{Conclusiones}

A lo largo de este artículo, hemos indagado en cómo todas aquellas actividades cotidianas que damos por sentado pueden entregarnos pistas de concepciones más amplias de la manera en que habitamos y construimos nuestras ciudades. Inspirados por el llamado de George Perec a interrogar lo cotidiano, desarrollamos la idea de que la ropa tendida puede ser comprendida como una multiplicidad de gestos. Los gestos, en cuanto movimientos portadores y productores de significado, nos invitan a develar su significado y a preguntarnos por lo que hay detrás de ellos; así identificamos tres gestos en la ropa tendida: el material, el político y el poético.

Nuestros esfuerzos aquí no pretenden ser exhaustivos, sino más bien plantear una invitación a repensar aquellas prácticas cotidianas de nuestra vida en cuanto portadoras y creadoras de sentido. En última instancia, nuestro interés está enmarcado por la comprensión de que incluso las actividades más banales son parte importante de nuestro hacer cotidiano y, por lo tanto, son capaces de afectarnos y de movernos en maneras sutiles, pero persistentes. Nos parece acertado incluir aquí un fragmento de libro de Kathleen Stewart, Ordinary affects, que justamente refiere a esta capacidad de lo cotidiano: 
Lo ordinario es una cosa que hay que imaginar y habitar.

También es una conexión sensorial. Un salto.

$Y$ un mundo de afinidades e impactos que tienen lugar en movimientos de intensidad a través de cosas que parecen sólidas y muertas (...).

La vaguedad de la calidad inacabada de lo ordinario no es tanto una deficiencia como un recurso, como una niebla de fuerzas inmanentes que aún se mueven a pesar de que ya han pasado muchas cosas y parece que hay muchas cosas que están grabadas en piedra.

Esto no es una utopía. No es un desafío que alcanzar o un ideal que se debe realizar, sino un modo de sintonía que responde de manera continua a algo que todavía no se ha dado y que de alguna manera está sucediendo (Stewart 2007, 127, traducción propia).

Los gestos de la ropa tendida aquí descritos implican que esta funciona en la urbe como una intervención sensible, es decir, como intervención que capta nuestra atención apelando a nuestros sentidos. En esa simple condición, la ropa tendida nos hacer ver y nos interpela. La ropa tendida "aparece" en un lugar y, con su aparición, activa un modo de vinculación en el que emergemos como copresentes, como observadores y como potenciales productores de enunciados sobre lo observado, ya sea en alguno de los registros gestuales recién evocados o en otros por descubrir.

\section{Bibliografía}

Appadurai, Arjun. 1986. The social life of things: Commodities in cultural perspective. Cambridge: Cambridge Cambridge University Press, 1986.

Back, Les. 2015. «Why Everyday Life Matters: Class, Community and Making Life Livable». Sociology. 49(5): https://doi.org/10.1177/0038038515589292.

Banerjee, Mukulika. y Daniel Miller. 2003. The Sari. Oxford: Berg, 2003. 
Bardet, Marie. 2018. «Saberes gestuales Epistemologías, estéticas y políticas de un «cuerpo danzante»». Enrahonar. 60(2), 13-28.

Bayly, Christopher. 1986. "The Origins of Swadeshi: cloth and Indian Society», 1700-1930. En The Social Life of Things, editado por Arjun Appadurai, 285-322. Cambridge: Cambridge University Press.

Courpasson, David. 2017. «The Politics of Everyday». Organization Studies. 38(6), 843-859. https://doi.org/10.1177/0170840617709310

Dant, Tim. 1999. Material culture in the social world: Values, activities, lifestyles. Philadelphia, PA: Open University Press.

de Certeau, Michel. 1979. La invención de lo cotidiano. México: Universidad Iberoamericana.

Flusser, Vilem. 2014. Gestures. Minneapolis: University of Minnesota.

Garfinkel, Harold. 1967. Studies in Ethnomethodology. Englewood Cliffs, NJ: Prentice-Hall.

Goffman, Erwin. 1959. The presentation of self in everyday life. New York: Doubleday Anchor Books.

Gregson, Nicky, y Vikki Beale. 2004. «Wardrobe matter: The sorting, displacement and circulation of women's clothing». Geoforum. 35(6), 689-700. https://doi.org/10.1016/j.geoforum.2004.03.014.

Gullestad, Marianne. 1995. «Home decoration as popular culture: constructing homes, genders and classes in Norway». En The Politics of Domestic Consumption: critical readings, editado por Stevi Jackson y Shaun Moores, 321-335. London: Routledge.

Hernández García, Iliana, Raúl Niño Bernal, y Jaime Hernández-García. 2018. Ecopolítica de los paisajes artificiales. Bogotá: Editorial Pontificia Universidad Javeriana.

Hernández GarciaGarcía, Jaime. 2013. Construcción social de espacio público en barrios populares de Bogotá. Revisa INVI, 28(78), 143-178.

Highmore, Ben. 2018. «Georges Perec and the Significance of the Insignificant». En The afterlives of Georges Perec, editado por Rowan Wilken y Justin Clemens, 105-119. Edinburgh: Edinburgh University Press.

Ingold, Tim. 2011. The perception of the environment: Essays on livelihood, dwelling and skill. London: Routledge.

Küchler, Susanne, y Daniel Miller (Edseds.). 2005. Clothing as Material Culture. Oxford: Berg.

Lefebvre, Henri y Catherine Régulier. 1985. "Le projet rythmanalytique». Communications. 41: 191-199. 
Margiotti, Margherita. 2013. «Clothing sociality: Materiality and the everyday among the Kuna of Panama». Journal of Material Culture. 18(4): 389407. https://doi.org/10.1177/1359183513502263

Martínez, Ion. 2013. «Henri Lefebvre y los espacios posibles». En La producción del espacio, escrito por Henri Lefebvre, 9-28. Madrid: Capitán Swing.

Massey, Doreen. 2005. Space, place, and gender. Minneapolis: University of Minnesota Press.

Miller, Daniel. 2005. Acknowledging consumption: A review of new studies. London: Routledge.

Noland, Carrie. 2009. Agency and embodiment: Performing gestures/producing culture. Cambridge, MA: Harvard University Press.

Norris, Lucy. 2004. "Shedding skins: The materiality of divestment in India». Journal of Material Culture. 9(1): 59--71.

Perec, George. 2008. Lo infraordinario. Impedimenta.

Pink, Sarah. 2012. Situating everyday life. Los AngelesÁngeles, CA, London: SAGE.

Rancière, Jacques. 2009. El reparto de lo sensible: Estética y política. Santiago: LOM.

Rancière, Jacques. 2020. Le temps du paysage. Aux origines de la révolution esthétique. París: La Fabrique.

Robinson, Jennifer. 2006. Ordinary cities: Between modernity and development. London: Routledge.

Roy, Ananya. 2009. "The 21st-Century Metropolis: New Geographies of Theory». Regional Studies. 43(6): 819-830. https://doi.org/10.1080/00343400701809665

Sheringham, Michael. 2000. «Attending to the everyday: Blanchot, Lefebvre, Certeau, Perec». French Studies, 54: 187--199.

Silva, Armando. 1992. Imaginarios urbanos: cultura y comunicación urbana. Bogotá: Tercer Mundo Editores.

Silva, Rebeca, Francisco Muñoz, y Hugo Delanoë. 2016. «Ergociudad. Hacia la producción relacional del espacio urbano». Revista de Geografía Espacios 6(11): 15-31.-

Stewart, Kathleen. 2007. Ordinary affects. Duke University Press.

Weiner, Annette B. y Jane Schneider. (Edseds.) 1989. Cloth and the Human Experience. London: Smithsonian Institute Press.

Wright Mills, Charles. 1959. The Sociological Imagination. Oxford: Oxford University Press. 